\title{
ATIVIDADES DESENVOLVIDAS POR PROFISSIONAIS DE NÚCLEOS DE APOIO À SAÚDE DA FAMÍLIA: REVISÃO DA LITERATURA
}

\author{
ACTIVITIES DEVELOPED BY FAMILY HEALTH SUPPORT CENTER \\ PROFESSIONALS: REVIEW OF THE LITERATURE
}

\author{
ACTIVIDADES LLEVADAS A CABO POR PROFESIONALES DE NÚCLEOS DE \\ APOYO A LA SALUD DE LA FAMILIA: REVISIÓN DE LA LITERATURA
}

\author{
Vladimir Andrei Rodrigues Arce ${ }^{1}$ \\ Carmen Fontes Teixeira ${ }^{2}$
}

Resumo Este artigo tem como objetivos sintetizar as características da produção científica brasileira acerca das atividades desempenhadas pelos profissionais dos Núcleos de Apoio à Saúde da Família, analisar o conteúdo destas atividades e identificar os aspectos facilitadores e dificultadores do processo de trabalho da equipe. Tratase de uma revisão da literatura publicada no período de 2008 a 2015 nas bases eletrônicas SciELO e Lilacs. Os resultados evidenciam a predominância de estudos qualitativos que descrevem as atividades realizadas por profissionais específicos, especialmente o acolhimento, a assistência e a reabilitação, sendo menos expressivas as ações de promoção da saúde, prevenção, proteção e vigilância, planejamento, programação, controle, avaliação e gestão. As ações de capacitação e educação permanente são as menos discutidas. São aspectos facilitadores a proatividade e a boa relação interprofissional, e dificultadores a persistência do modelo biomédico no âmbito da Saúde da Família, as precárias condições de trabalho e a falta de apoio da gestão. Percebe-se uma tendência à reprodução da perspectiva assistencial hegemônica no âmbito do Núcleo de Apoio à Saúde da Família, em detrimento de um processo de mudança orientado para a integralidade da atenção.

Palavras-chave saúde da família; atenção primária à saúde; prática de saúde pública; políticas públicas de saúde; equipe de assistência ao paciente.
Abstract The goals of this article are to summarize the characteristics of the Brazilian scientific production regarding the activities performed by the professionals of the Family Health Support Centers, to analyze the content of those activities, and to identify the facilitating and hindering aspects in the teams' work process. We performed a review of the literature published between 2008 and 2015 in the SciELO e LILACS electronic databases. The results evince the prevalence of qualitative studies that describe the activities performed by specific professionals, particularly the user embracement, the care and the rehabilitation, with the actions of promotion of health, prevention, protection and vigilance, planning, programming, control, evaluation and management being less expressive. The training and permanent education actions are the least discussed actions. Among the facilitating aspects are proactivity and good interprofessional relationship, and among the hindering aspects are the persistence of the biomedical model within the Health of the Family field, the precarious working conditions, and the lack of support on the part of the management. We noticed a tendency to reproduce the hegemonic care perspective within the Health of the Family Support Centers, to the detriment of a process of change geared towards the comprehensiveness of care.

Keywords family health; primary health care; public health practice; public health policies; patient care team. 


\section{Introdução}

Os Núcleos de Apoio à Saúde da Família (Nasfs) foram criados com o objetivo de ampliar a abrangência das ações das equipes de Saúde da Família (eSF) com vistas ao fortalecimento da integralidade da atenção, não só apoiando ações já existentes mas também desenvolvendo novas abordagens no território, quando necessário (Brasil, 2008a), o que implicou a incorporação de várias categorias profissionais à Atenção Primária à Saúde (APS) no Sistema Único de Saúde (SUS).

As atividades previstas para esses profissionais, embora sejam atravessadas pelas características gerais do trabalho na Estratégia Saúde da Família (ESF), fundamentam-se principalmente na ideia do apoio matricial (Oliveira e Campos, 2015; Cunha e Campos, 2011; Campos e Domitti, 2007). O apoio matricial visa à superação da lógica fragmentada de encaminhamentos clínicos entre os profissionais, por meio de retaguarda assistencial e apoio técnico-pedagógico às eSF. Trata-se de um mecanismo privilegiado de educação permanente, pautado na interprofissionalidade, no trabalho em redes, na atuação em território definido, no compartilhamento de saberes, na deliberação conjunta e na cogestão, além de um componente educador e formativo, por proporcionar espaços de discussão, troca de saberes e reflexão para a prática (Bispo Júnior e Moreira, 2017).

Diante desse novo contexto na APS brasileira, o debate em torno do processo de trabalho em saúde na ESF, questão crucial para o seu fortalecimento (Shimizu e Carvalho Junior, 2012; Reis et al., 2007; Ribeiro, Pires e Blank, 2004), tem se revigorado e mobilizado profissionais, gestores, pesquisadores e docentes da área de saúde para a reflexão sobre as repercussões da inclusão do Nasf no SUS.

Analisar as atividades que vêm sendo desenvolvidas no âmbito do Nasf pode ser útil para a identificação das características que marcam o processo de implantação e desenvolvimento desta proposta no Brasil, permitindo adequações e mudanças em pontos frágeis, ou mesmo manutenção e aprofundamento de potencialidades, de modo que o propósito de fortalecimento da APS se concretize. Nesse sentido, este artigo busca sintetizar as características da produção científica sobre as atividades desempenhadas pelos profissionais dos Nasf, bem como analisar o conteúdo destas atividades, identificando tendências atuais e aspectos que facilitam ou dificultam o processo de trabalho que vem sendo construído em diferentes contextos no Brasil.

\section{Metodologia}

Trata-se de um estudo de revisão de literatura em que se buscou levantar os artigos sobre as diferentes atividades desempenhadas pelos profissionais dos 
Nasfs publicados desde a criação destes até 2015, tendo como fonte de dados as bases eletrônicas SciELO e Lilacs.

Considerando-se que o Nasf é uma proposta relativamente recente no cenário da APS no Brasil, optou-se por adotar o uso dos termos "Núcleo(s) de Apoio à Saúde da Família" e "Nasf", relacionados por meio do operador booleano OR, de forma a viabilizar um levantamento mais abrangente de artigos relacionados ao tema. Na base de dados SciELO, a pesquisa foi feita considerando-se os filtros "todos os índices" e "português". Na base de dados Lilacs, acessada por meio do portal da Biblioteca Virtual em Saúde (BVS), utilizaram-se os filtros "português", "texto completo" e "artigo".

Inicialmente foram levantados 402 artigos na SciELO e 548 artigos na Lilacs, totalizando 950 documentos. Foram analisados os títulos e os resumos desses trabalhos, adotando-se como critério de inclusão a abordagem, com base em análises empíricas, das atividades desenvolvidas no âmbito do Nasf, sendo considerados tanto os trabalhos que tinham como objeto específico as atividades do Nasf, o que inclui abordagens de categorias específicas, quanto trabalhos voltados para as atividades gerais desenvolvidas por quaisquer profissionais da ESF, porém com a atuação de profissionais desse núcleo explicitamente destacada no texto. Assim, foram incluídos os artigos de caráter avaliativo e os relatos de experiência, tendo sido depurados os artigos que abordavam outras temáticas, os artigos de natureza teórica ou ensaística e as revisões de literatura referentes ao Nasf, o que resultou na seleção de 71 artigos da SciELO e 50 da Lilacs, totalizando 121 trabalhos. Foram excluídas as duplicações, resultando um número de 56 artigos, os quais foram lidos na íntegra, sendo excluídos outros 4 referentes a resumos de dissertações ou teses e ainda artigos que tinham como foco algum instrumento de trabalho muito específico do Nasf, como protocolos de avaliação clínica, restando, portanto, 52 artigos. Verificou-se ainda que 8 artigos não discriminavam as atividades dos profissionais no corpo do texto, embora abordassem de maneira genérica o trabalho no Nasf, tendo sido, portanto, excluídos, o que resultou em um total de 44 artigos analisados.

Inicialmente foi feita uma análise bibliométrica (Vosgerau e Romanowski, 2014) dos artigos segundo: ano de publicação; região geográfica de afiliação institucional dos autores; área do conhecimento e revista - segundo classificação da Coordenação de Aperfeiçoamento de Pessoal de Nível Superior (Capes); metodologia e abrangência do estudo; fontes de dados utilizadas; agente do trabalho enfocado no estudo (trabalho da equipe ou trabalho de uma profissão específica); e área temática central, considerando-se as áreas estratégicas definidas pelo Ministério da Saúde (Brasil, 2008b). Posteriormente, foi feita a análise de conteúdo dos artigos e definidas três categorias operacionais apriorísticas: Atividades desenvolvidas no âmbito do Nasf; Aspectos facilitadores do trabalho no Nasf); e Aspectos dificultadores do trabalho no Nasf. 
As atividades foram analisadas de acordo com cinco subcategorias previamente construídas com base nas contribuições teóricas de Teixeira e Vilasbôas (2014), Almeida-Filho (2011) e Paim (2006), a saber: Atividades de planejamento, programação, controle, avaliação e gestão; Atividades de promoção da saúde, atuação intersetorial e ações sobre o território; Atividades de prevenção de doenças, proteção e vigilância; e Atividades de acolhimento, assistenciais e de reabilitação/tratamento. Além dessas, os dados produzidos tornaram necessário construir uma quinta subcategoria: Atividades de capacitação e educação permanente.

Os aspectos facilitadores e dificultadores do trabalho, por sua vez, foram organizados também em subcategorias, baseadas nos elementos que emergiram das informações obtidas nos artigos.

\section{Panorama das atividades desenvolvidas nos Núcleos de Apoio à Saúde da Família no Brasil}

Os resultados da análise bibliométrica evidenciaram que os primeiros artigos foram publicados a partir de 2010 (2 artigos), tendo havido um crescimento importante no volume das publicações a partir de 2013 (13 artigos), com pico de produção em 2015 (15 artigos). Essas publicações estão concentradas basicamente em duas regiões do país: Sudeste e Nordeste, observando-se grande concentração da produção científica no estado de São Paulo. Há pouca contribuição entre pesquisadores de diferentes regiões do país: 3 artigos, 1 envolvendo Nordeste e Sudeste, 1 envolvendo Nordeste e Sul e 1 envolvendo Sul e Sudeste. Além disso, registrou-se apenas 1 produção oriunda de parceria internacional, especificamente com pesquisadores do Canadá (Tabela 1).

Tabela 1

Distribuição de artigos segundo localização da instituição dos autores

\begin{tabular}{llcc}
\hline \multicolumn{1}{l}{ Localização da instituição dos autores } & Artigos & \% \\
\hline Região & Estado & $\mathbf{n}$ & 0 \\
Norte & & 0 & 9,1 \\
Nordeste & Ceará & 4 & 6,8 \\
& Pernambuco & 3 & 6,8 \\
& Paraíba & 3 & 4,5 \\
& Rio Grande do Norte & 2 & 11,4 \\
& Outros estados & 5 & $\mathbf{3 8 , 6}$ \\
Sul & Subtotal & $\mathbf{1 7}$ & 4,5 \\
& Paraná & 2 & $\mathbf{4 , 5}$ \\
Sudeste & Subtotal & $\mathbf{2}$ & 27,3 \\
& São Paulo & 12 & 11,4 \\
& Minas Gerais & 5 & 4,5 \\
Centro-Oeste & Rio de Janeiro & 2 & $\mathbf{4 3 , 2}$ \\
& Subtotal & $\mathbf{1 9}$ & 2,3 \\
Colaboração inter-regional & Subtotal & 1 & 2,3 \\
Colaboração internacional & & 1 & $\mathbf{4 , 6}$ \\
\hline Total & & $\mathbf{2}$ & $\mathbf{6 , 8}$ \\
\hline
\end{tabular}

Fonte: Os autores. 
Tomando-se como referência as áreas do conhecimento da Coordenação de Aperfeiçoamento de Pessoal de Nível Superior (Capes), observa-se o predomínio de artigos publicados na grande área Ciências da Saúde, com poucas publicações na grande área Ciências Humanas. Considerando-se as áreas específicas, verifica-se que aproximadamente metade $(47,7 \%)$ foi veiculada em periódicos da Saúde Coletiva, sendo que $20,4 \%$ da produção foram publicados em revistas de Educação Física (que inclui Educação Física, Fisioterapia, Fonoaudiologia e Terapia Ocupacional) e 13,6\% na área de Psicologia (Tabela 2).

Tabela 2

\begin{tabular}{|c|c|c|c|c|}
\hline $\begin{array}{c}\text { Área do } \\
\text { conhecimento }\end{array}$ & Áreas específicas & Revista científica & $\mathbf{n}$ & $\%$ \\
\hline \multirow[t]{26}{*}{ Ciências da Saúde } & Saúde Coletiva & Ciênçia \& Saúde Coletiva & 5 & 11,4 \\
\hline & & Physis Revista de Saúde Coletiva & 4 & 9,1 \\
\hline & & Saúde em Debate & 3 & 6,7 \\
\hline & & Interface & 2 & 4,5 \\
\hline & & Revista Brasileira de Promoção da Saúde & 2 & 4,5 \\
\hline & & Revista APS & 1 & 2,3 \\
\hline & & Trabalho, Educação e Saúde & 1 & 2,3 \\
\hline & & Revista de Saúde Pública & 1 & 2,3 \\
\hline & & Revista Brasileira de Saúde Ocupacional & 1 & 2,3 \\
\hline & & Epidemiologia e Serviços de Saúde & 1 & 2,3 \\
\hline & & Subtotal & 21 & 47,7 \\
\hline & Educação Física & Codas & 2 & 4,5 \\
\hline & & Cadernos de Terapia Ocupacional da Ufscar & 2 & 4,5 \\
\hline & & Cefac & 2 & 4,5 \\
\hline & & Audiology Communication Research & 1 & 2,3 \\
\hline & & Fisioterapia em Movimento & 1 & 2,3 \\
\hline & & Revista Brasileira de Atividade Física e Saúde & 1 & 2,3 \\
\hline & & Subtotal & 9 & 20,4 \\
\hline & Enfermagem & Revista de Pesquisa Cuidado é Fundamental & 2 & 4,5 \\
\hline & & Escola Anna Nery & 1 & 2,3 \\
\hline & & Texto e Contexto - Enfermagem & 1 & 2,3 \\
\hline & & Subtotal & 4 & 9,1 \\
\hline & Nutrição & $\begin{array}{l}\text { Revista de Nutrição } \\
\text { Subtotal }\end{array}$ & $\begin{array}{l}2 \\
2\end{array}$ & $\begin{array}{l}4,5 \\
\mathbf{4 , 5}\end{array}$ \\
\hline & Inespecífica & O Mundo da Saúde & 1 & 2,3 \\
\hline & & Revista Brasileira de Ciências da Saúde & 1 & 2,3 \\
\hline & & Subtotal & 2 & 4,6 \\
\hline \multirow[t]{4}{*}{ Ciências Humanas } & Psicologia & Psicologia USP & 2 & 4,5 \\
\hline & & Psicologia, Ciência e Profissão & 2 & 4,5 \\
\hline & & Estudos de Psicologia & 1 & 2,3 \\
\hline & & $\begin{array}{l}\text { Psicologia e Sociedade } \\
\text { Subtotal }\end{array}$ & $\begin{array}{l}1 \\
6\end{array}$ & 2,3 \\
\hline Total & & & 44 & 100 \\
\hline
\end{tabular}

Em relação aos aspectos metodológicos dos artigos, percebe-se amplo predomínio de desenhos do tipo qualitativo, correspondentes a 77\% (34 artigos), aos quais se somam 3 que integraram técnicas qualitativas e quantitativas. $54 \%$ das análises foram realizadas no âmbito municipal (24 artigos), sendo que $20 \%$ (9 artigos) consideraram o contexto local de atuação das equipes Nasf e 14\% (6 artigos), os distritos sanitários. As menores frequências estão relacionadas às abrangências estadual e nacional, com 7\% (3 artigos) e 5\% (2 artigos), respectivamente. 
Analisando-se as fontes de dados acessadas nessas publicações, verificou-se que a quase totalidade dos artigos (98\%) fez uso de dados primários. Salienta-se que 4 artigos (9\%) fizeram uso de ambas as fontes de dados, e apenas 1 artigo utilizou somente dados secundários, a exemplo de relatórios e documentos de credenciamento das equipes no Ministério da Saúde (MS).

Os profissionais do Nasf foram sujeitos da pesquisa em $89 \%$ do total de produções (39 artigos), sendo que os profissionais das eSF foram público-alvo das pesquisas em apenas 8 artigos, mesmo número de estudos que envolveram os gestores. Além disso, verificou-se que a maioria dos estudos $(56,8 \%)$ abordou as práticas do Nasf com foco em uma categoria profissional específica, em detrimento da perspectiva de equipe.

Ressalta-se ainda que 12 artigos consideraram mais de um tipo de sujeito na composição dos públicos-alvo de suas pesquisas, tendo sido possível identificar 7 diferentes categorias profissionais, destacando-se o psicólogo e o fonoaudiólogo. Apenas um artigo enfocou o trabalho do médico, especificamente o psiquiatra. Os usuários compuseram o grupo menos estudado, tendo sido incluídos em apenas 2 artigos (4,5\%) (Tabela 3).

Tabela 3

\begin{tabular}{llcc}
\hline Distribuição de artigos segundo agente do trabalho enfocado nos estudos & & \\
\hline Agente do trabalho enfocado & & $\mathbf{n}$ & $\%$ \\
\hline Profissional específico & Psicólogo & 7 & 16 \\
& Fonoaudiólogo & 5 & 11,3 \\
& Nutricionista & 4 & 9,1 \\
& Terapeutas ocupacionais & 3 & 6,8 \\
& Fisioterapeuta & 3 & 6,8 \\
& Profissional de educação física & 2 & $\mathbf{4 , 5}$ \\
Equipe Nasf & Médico psiquiatra & 1 & $\mathbf{2 , 3}$ \\
\hline Total & Subtotal & $\mathbf{2 5}$ & $\mathbf{5 6 , 8}$ \\
\hline Fonte: Os autores. & & $\mathbf{1 9}$ & $\mathbf{4 3 , 2}$ \\
\hline
\end{tabular}

Quanto às áreas temáticas, verificou-se que apenas 6 artigos abordaram as áreas temáticas específicas propostas pelo MS, tendo sido possível identificar as seguintes áreas: Saúde Mental (2 artigos); Reabilitação; Alimentação e Nutrição; Saúde da Mulher; e Saúde do Idoso (1 artigo cada). A maioria da produção $(81,38 \%, 36$ artigos) enfocou elementos gerais do processo de trabalho em saúde no âmbito do Nasf, ou seja, buscavam fazer análises do trabalho como um todo desenvolvido nos núcleos. Integram esse grupo 3 artigos que, ao abordarem o trabalho em geral no Nasf, partiram da categoria apoio matricial, o que não foi observado nos demais.

Ressalta-se ainda que 6 dos artigos que discutiram o processo de trabalho em geral do Nasf também abordaram, concomitantemente, o processo de implantação ( 3 artigos) e a formação dos profissionais do Nasf ( 3 artigos). Apenas 2 artigos enfocaram as práticas de promoção da saúde e gestão (1 cada). 


\section{Análise do conteúdo dos artigos selecionados}

As principais características das atividades desenvolvidas pelos profissionais relatadas na literatura estão sintetizadas nas cinco subcategorias definidas (Quadro 1). Predominaram referências a "atividades de acolhimento, assistenciais e de reabilitação/tratamento", únicas a serem tratadas em todos os artigos. Em seguida, embora menos expressivas quando comparadas às primeiras, destacaram-se as "atividades de prevenção de doenças, proteção e vigilância", bem como as "atividades de planejamento, programação, controle, avaliação e gestão". As "atividades de promoção da saúde, atuação intersetorial e ações sobre o território", por sua vez, foram pouco expressivas nos artigos, e as menos referidas foram as "atividades de matriciamento e capacitação/educação permanente de profissionais".

\begin{tabular}{|c|c|}
\hline \multicolumn{2}{|c|}{ Características das atividades dos profissionais do Nasf descritas na literatura } \\
\hline Subcategoria & Eixos \\
\hline $\begin{array}{l}\text { Atividades } \\
\text { Atividades de acolhimento, } \\
\text { assistenciais e de reabilitação/ } \\
\text { tratamento }\end{array}$ & $\begin{array}{l}\text { Atendimento individual (específico ou compartilhado) ao usuário } \\
\text { Atendimentos domiciliares } \\
\text { Grupos de avaliação clínica e grupos terapêuticos } \\
\text { Acolhimento dos usuários } \\
\text { Ações de cuidado em saúde voltadas para os trabalhadores da Estratégia Saúde da } \\
\text { Família }\end{array}$ \\
\hline $\begin{array}{l}\text { Atividades de prevenção de } \\
\text { doenças, proteção e vigilância }\end{array}$ & Ações educativas e sensibilizadoras de prevenção \\
\hline $\begin{array}{l}\text { Atividades de planejamento, } \\
\text { programação, controle, avaliação } \\
\text { e gestão }\end{array}$ & $\begin{array}{l}\text { Apoio à gestão dos sistemas e serviços de saúde } \\
\text { Planejamento e gestão do próprio trabalho } \\
\text { Análise de situação de saúde e das demandas das equipes de Saúde da Família } \\
\text { Avaliação do trabalho }\end{array}$ \\
\hline $\begin{array}{l}\text { Atividades de promoção da saúde, } \\
\text { atuação intersetorial e ações } \\
\text { sobre o território }\end{array}$ & $\begin{array}{l}\text { Territorialização e construção de redes intersetoriais de apoio } \\
\text { Práticas de promoção da saúde } \\
\text { Comunicação das atividades na comunidade }\end{array}$ \\
\hline $\begin{array}{l}\text { Atividades de capacitação } \\
\text { e educação permanente de } \\
\text { profissionais }\end{array}$ & Ações de capacitação dos profissionais das equipes de Saúde da Família \\
\hline Facilidades & $\begin{array}{l}\text { Perfil proativo e boa relação estabelecida entre os profissionais que compõem o Nasf } \\
\text { Características contra-hegemônicas da proposta organizacional oficial do Nasf } \\
\text { Apoio da gestão } \\
\text { Bom funcionamento das unidades de saúde e o apoio da rede assistencial e } \\
\text { intersetorial } \\
\text { Qualificação dos profissionais } \\
\text { Apoio das equipes de Saúde da Família }\end{array}$ \\
\hline Dificuldades & $\begin{array}{l}\text { Hegemonia do modelo de atenção biomédico e a resistência das equipes de Saúde } \\
\text { da Família à proposta do Nasf } \\
\text { Falta de apoio da gestão e más condições de trabalho } \\
\text { Formação, qualificação e perfil profissional pouco adequados à proposta } \\
\text { Desestruturação das redes de atenção à saúde e grande demanda reprimida }\end{array}$ \\
\hline
\end{tabular}

Em relação à subcategoria "atividades de acolhimento, assistenciais e de reabilitação/tratamento", foi possível identificar cinco eixos. O primeiro eixo, que se refere ao "atendimento individual (específico ou compartilhado) ao usuário", foi o mais frequentemente descrito nos estudos, e diz respeito ao atendimento clínico-assistenciais aos sujeitos que apresentam algum tipo de patologia/doença e/ou sofrimento que esteja afetando sua saúde. Esse atendimento pode ser feito por apenas um profissional do Nasf ou por meio de 
uma intervenção compartilhada entre profissionais das eSF e profissionais do Nasf. Há pouco detalhamento sobre tais atividades, e apenas um artigo faz referência a um atendimento clínico orientado por uma perspectiva teórica específica, a reabilitação baseada na comunidade.

O segundo eixo é referente a "atendimentos domiciliares", em geral relacionados a sujeitos acometidos por problemas de saúde que estejam com dificuldades para acessar o serviço de saúde, caracterizando uma expansão do atendimento ambulatorial que termina por permitir ao profissional conhecer melhor a realidade em que tais sujeitos vivem. O terceiro eixo diz respeito às atividades desenvolvidas em "grupos de avaliação clínica e grupos terapêuticos", nos quais se busca ampliar a abrangência das ações de cunho assistencial para um maior número de pessoas, porém há poucas informações sobre os aspectos teórico-metodológicos que sustentam as abordagens grupais. Menos expressivas, as atividades de "acolhimento dos usuários" nos serviços de saúde compõem o quarto eixo, seja quando os profissionais integram programas de acolhimento da unidade, seja quando acolhem usuários direcionados ao Nasf. E as "ações de cuidado em saúde aos trabalhadores da $\mathrm{ESF}^{\prime}$, que se referem principalmente às atividades voltadas para o cuidado à saúde dos agentes comunitários de saúde, dizem respeito ao quinto e último eixo e aparecem em apenas 2 artigos.

As "atividades de prevenção de doenças, proteção e vigilância", por sua vez, têm um único eixo, que se refere às "ações educativas e sensibilizadoras de prevenção", incluindo atividades desenvolvidas em oficinas, grupos, salas de espera e palestras, geralmente relacionadas aos conhecimentos específicos dos diferentes profissionais do Nasf. Embora não haja detalhamento de tais atividades, transparece a ênfase na adoção de práticas centradas na mudança de comportamentos dos usuários. Neste eixo é possível verificar a execução de ações programáticas, a partir da inserção dos profissionais em programas de saúde pública já existentes nas unidades, tais como ações voltadas para usuários com hipertensão e diabetes, dentre outros, além de ações de vigilância. Ademais, atividades de produção de material educativo fazem parte deste eixo.

A terceira subcategoria observada é concernente às "atividades de planejamento, programação, controle, avaliação e gestão", bem menos expressivas que as atividades assistenciais, sendo o eixo do "apoio à gestão dos sistemas e serviços de saúde" o mais evidente, envolvendo tanto ações nas unidades de saúde quanto ações mais complexas no nível da gestão municipal, como é o caso da implantação de programas de avaliação e qualificação da APS, bem como a participação em áreas temáticas/linhas de cuidado estruturadas nas secretarias municipais de Saúde. O eixo "planejamento e gestão do próprio trabalho", também nesta categoria, evidencia as ações dos profissionais do Nasf voltadas para a organização do cotidiano de trabalho, que envolve a criação de rotinas e protocolos assistenciais, fluxos e confecção de relatórios e fichas 
de produção, bem como a pactuação permanente de agendas e ações com as eSF. O eixo das atividades de "análise de situação de saúde e das demandas das equipes de Saúde da Família" e o eixo das atividades de "avaliação do trabalho" realizado pela equipe ocupam um espaço pouco relevante no contexto geral do trabalho do núcleo.

As "atividades de promoção da saúde, atuação intersetorial e ações sobre o território" não obtiveram destaque entre as abordadas nos diferentes estudos analisados. Ainda assim, foi possível identificar três eixos que caracterizam tais atividades. O primeiro eixo, referente à "territorialização e construção de redes intersetoriais de apoio", evidencia o processo de articulação dos profissionais do Nasf com demais equipamentos sociais no território em que estão inseridos. Embora o detalhamento dessas atividades seja insuficiente, essa articulação parece ocorrer principalmente com instituições do setor público, a exemplo dos Centros de Referência da Assistência Social (CRASs), havendo pouca referência a movimentos sociais ou lideranças comunitárias.

O segundo eixo diz respeito às "práticas de promoção da saúde", por meio de ações educativas em grupos e oficinas, geralmente lúdicas, tais como passeios e bailes. Também se destacam as atividades relacionadas aos equipamentos educacionais, como escolas e creches. O último eixo, referido apenas por 2 artigos, diz respeito à "comunicação das atividades na comunidade", evidenciando a incipiência da comunicação direta e permanente do Nasf com a população do território que não está em atendimento direto na unidade de saúde, a exemplo de ações de divulgação das atividades desenvolvidas pelos profissionais em espaços comunitários.

Por fim, a última subcategoria identificada é referente às "atividades de capacitação e educação permanente de profissionais". Chama a atenção o fato de tais atividades serem pouco referidas nos estudos analisados, aparecendo apenas menções a "ações de capacitação dos profissionais das equipes de Saúde da Família", em diversos temas, como Saúde Mental, Alimentação e Nutrição e Humanização, com escassa descrição das metodologias abordadas, havendo referências tanto ao uso de ferramentas tradicionais de formação continuada, como palestras, quanto à articulação entre o processo formativo e o processo assistencial vivido no trabalho, o que caracterizaria a educação permanente.

Também foram identificadas referências, ainda que com pouca expressão, às atividades de "discussão de casos", que ocorrem sobretudo nas reuniões do Nasf com as eSF e, em geral, estão relacionadas à construção do projeto terapêutico singular dos diferentes casos discutidos. Optou-se por não inserir tais atividades em nenhuma das categorias anteriores porque não foi possível classificar se estão relacionadas ao processo assistencial, ao matriciamento das equipes, ou a ambos. $\mathrm{O}$ mesmo ocorreu em relação às reuniões, tanto as que são feitas com as eSF quanto as do próprio Nasf. Mesmo que bastante citadas como atividades estruturantes do trabalho do Nasf, a maioria dos artigos não 
explicitava claramente a finalidade das reuniões, o que dificultou o processo de categorização.

\section{Facilidades e dificuldades enfrentadas pelos profissionais do Nasf}

Observou-se predomínio de referências às dificuldades, sendo que 14 artigos sequer abordaram os aspectos considerados facilitadores do trabalho. De todo modo, foi possível identificar 6 aspectos que facilitam o trabalho no Nasf.

$\mathrm{O}$ primeiro aspecto facilitador diz respeito ao "perfil proativo e à boa relação estabelecida entre os profissionais do Nasf", evidenciando que parte importante do êxito do trabalho se deve ao empenho e à capacidade de entrosamento desses profissionais, o que é demonstrado por meio de características subjetivas e habilidades relacionais descritas como disponibilidade, flexibilidade, postura acolhedora e capacidades de mobilização, adaptação e reflexão crítica.

O segundo está relacionado às "características contra-hegemônicas da proposta organizacional oficial do Nasf", que funciona como uma ancoragem para os profissionais, na medida em que podem se orientar por tais características para resistir a pressões produtivistas. Assim, a perspectiva do trabalho integrado em equipe, a corresponsabilização, a coletividade, a diversidade, a autonomia e a relação comunicativa e desburocratizada são características que, ainda que presentes na realidade de todas as equipes, tendem a favorecer o andamento das atividades no contexto do Nasf.

$\mathrm{O}$ "apoio da gestão" configura o terceiro aspecto, citado em 5 artigos. Essa questão está diretamente ligada à relação positiva entre o trabalho e o apoio político e operacional do gestor, que ocorre por meio da disponibilização de recursos materiais, da educação permanente e do estabelecimento de apoiadores institucionais para o trabalho do Nasf, de modo a favorecer a implantação de uma proposta inovadora na ESF.

O quarto se refere ao "bom funcionamento das unidades de saúde e o apoio da rede assistencial e intersetorial" e indica que os aspectos estruturais do contexto onde estão inseridos os Nasfs afetam diretamente o trabalho, que é favorecido quando as unidades de saúde conseguem desenvolver de maneira satisfatória seu trabalho, o que é fomentado quando há redes de atenção à saúde estruturadas, com efetivas parcerias não só com outros serviços de saúde, mas também com demais equipamentos sociais do território, ou mesmo com instituições de ensino superior.

Os dois últimos, referentes à "qualificação dos profissionais" e ao "apoio das equipes de saúde da família", embora evidenciem importantes aspectos facilitadores do trabalho, foram pouco significativos no conjunto dos trabalhos analisados. 
Os aspectos considerados dificultadores do trabalho no Nasf, abordados por todos os artigos, foram classificados em quatro grupos. O mais referido diz respeito à "hegemonia do modelo de atenção biomédico e à resistência das equipes de Saúde da Família à proposta do Nasf", identificando-se questões relacionadas à organização do trabalho da equipe Nasf, ainda fortemente influenciada por um modelo clínico fragmentado, também reproduzido pelas eSF. Aparecem várias referências à desigualdade de relações de poder entre o Nasf e as eSF, destacando-se com frequência a dificuldade de adesão à proposta por parte dos médicos dessas equipes.

O segundo aspecto dificultador, também bastante mencionado nos artigos analisados, é a "falta de apoio da gestão e más condições de trabalho". Pesa sobre as equipes Nasf a pressão de parte dos gestores, tanto locais quanto municipais, pelo aumento da produtividade, medida em termos das ações realizadas. Ademais, quando não há essa pressão, é comum haver omissão em relação ao Nasf, que se reflete tanto na indefinição de diretrizes claras para o trabalho e na falta de política para o seu aprimoramento quanto na precarização das condições de trabalho, evidenciadas na falta de infraestrutura física e material adequada, em contratos precários, salários reduzidos e sobrecarga de trabalho, entre outros.

Dificuldades relacionadas à "formação, qualificação e perfil profissional pouco adequados à proposta" compõem o terceiro aspecto, que, embora não tão expressivo quanto os dois primeiros, expõe a dificuldade relacionada ao perfil do agente das práticas de saúde do Nasf, que, em geral, não foi formado para uma atuação interdisciplinar condizente com os pressupostos da APS e tampouco teve oportunidade de participar de ações de capacitação para tal no ambiente de trabalho.

O último aspecto é a "desestruturação das redes de atenção à saúde e grande demanda reprimida", que evidencia as dificuldades relacionadas ao contexto estrutural do trabalho, geralmente marcado por grande pressão, inclusive da população, por atendimentos clínicos para especialidades que não estão contempladas em outros níveis de atenção, fazendo com que o trabalho do Nasf tenha que responder majoritariamente a um propósito clínico reabilitador. A reduzida articulação com a rede intersetorial do território também é referida nesta categoria.

Além dessas dificuldades, foram feitas menções esparsas a outras barreiras encontradas no cotidiano das unidades de saúde, tais como dificuldade de acesso a prontuários e a falta de conhecimento, por parte de diferentes atores, sobre determinadas profissões. A situação de grande vulnerabilidade socioeconômica da população e a baixa participação desta na construção de propostas para o Nasf também foram questões apontadas como dificultadoras do trabalho do núcleo. 


\section{Sobre a necessidade de se ampliar as atividades profissionais no âmbito dos Nasfs em direção à integralidade}

Os dados obtidos na análise bibliométrica revelam que o interesse acadêmico sobre o trabalho no âmbito do Nasf vem ganhando corpo no contexto da pesquisa em APS, ainda que prevaleçam estudos de caráter descritivo e exploratório. A concentração atual das pesquisas em duas regiões, Sudeste e Nordeste, coincide com o fato de que estas são as regiões em que está a maior parte das equipes Nasf em todo o país (41\% no Nordeste e $29 \%$ no Sudeste), segundo dados do Cadastro Nacional de Estabelecimentos de Saúde (Brasil, 2016). Ademais, o crescimento também coincide com a publicação da portaria 3.124 do Ministério da Saúde (Brasil, 2012), que ampliou a possibilidade de credenciamento de novas equipes Nasf no país. O predomínio de pesquisas produzidas no estado de São Paulo, por sua vez, pode ser decorrente da concentração nesse estado do maior número de pesquisadores e grupos de pesquisa cadastrados no Diretório de Grupos de Pesquisa do Conselho Nacional de Desenvolvimento Científico e Tecnológico (CNPq, 2014).

O predomínio de estudos sobre o processo de trabalho em saúde dos profissionais do Nasf em relação aos demais enfoques específicos evidencia o interesse dos pesquisadores por um tema relevante no campo da saúde coletiva (Peduzzi e Schraiber, 2006), o que explica, em alguma medida, o fato de quase metade das publicações estarem veiculadas em revistas desta área. Entretanto, observa-se pouco investimento teórico, uma vez que prevalecem descrições e análises que tomam como base apenas as normas e diretrizes do Ministério da Saúde.

Ainda sobre as temáticas, não foram observados estudos de avaliação dos impactos da inserção do Nasf no trabalho das eSF, na (re)configuração das redes de atenção à saúde e na melhoria das condições de saúde dos usuários, pontos cruciais do processo de implantação e fortalecimento do SUS (Teixeira, Souza e Paim, 2014; Aquino et al., 2014; Paim et al., 2011; Barata, 2008).

Vale registrar que, embora o apoio matricial possa ser operacionalizado por meio de diferentes práticas no cotidiano do trabalho das equipes, poucos são os estudos que discutem especificamente o trabalho no Nasf tomando-o como referencial teórico, o que indica a necessidade de se investigar melhor como estão se configurando os processos de matriciamento nos territórios, bem como as potencialidades e limites dessa prática. Ademais, também são poucas as análises relacionadas a áreas definidas como prioritárias pelas diretrizes do MS para o Nasf (Brasil, 2008), tais como Saúde Mental, Reabilitação, Saúde da Mulher e Alimentação e Nutrição, não havendo pesquisas relacionadas diretamente às demais áreas, como Saúde da Criança e Práticas Integrativas, entre outras. Assim, faz-se necessário analisar se tais prioridades, sinaliza- 
das nacionalmente, coincidem ou não com as reais necessidades de saúde da população nos territórios.

O predomínio de estudos que focalizam o trabalho de profissionais específicos, a exemplo do psicólogo, do fonoaudiólogo ou do nutricionista, evidencia o interesse de pesquisadores de determinadas profissões em se debruçar sobre os diferentes aspectos do trabalho no âmbito da APS, o que tem sido observado também em outros países (Oliveira e Campos, 2015). Isso repercute no aumento observado de publicações relativas ao Nasf em revistas de áreas específicas, sinalizando maior integração de conhecimentos relacionados à APS em espaços acadêmicos geralmente restritos a assuntos especializados de diversas profissões.

Todavia, se por um lado esse recorte sinaliza uma possibilidade profícua para o surgimento de novas reflexões acerca das práticas de saúde e das identidades profissionais, por outro preocupa, na medida em que o olhar integral sobre o Nasf tem sido secundarizado. Tal secundarização demonstra que a perspectiva fragmentada do cuidado em saúde, característica do modelo médico hegemônico, ainda se reproduz em alguma medida no âmbito do Nasf, conforme observaram Arce e Teixeira (2017), o que precisa ser repensado.

Essa questão se torna especialmente preocupante nos primeiros anos de implementação de uma nova política, cujo desempenho depende não só da forma de execução de atividades como também das características das agências implementadoras e das condições políticas, econômicas e sociais (Viana, 1996). O predomínio do interesse pelo trabalho de profissionais específicos pode fragilizar uma perspectiva transformadora de modelo de atenção à saúde que tem como base a ideia de equipe como integração de trabalhos (Peduzzi, 2001), evidenciando a necessidade de maior investimento em pesquisas com enfoque no trabalho em equipe.

Em relação aos aspectos metodológicos, o amplo uso de métodos e técnicas qualitativas de produção e análise dos dados, associado a uma abrangência majoritariamente restrita ao nível municipal das pesquisas, reforça a percepção de que está em curso um importante movimento dos pesquisadores no sentido de compreender com maior profundidade as diferentes realidades e as especificidades que caracterizam o desenvolvimento do Nasf. A abordagem qualitativa, ao permitir uma aproximação fundamental entre sujeito e objeto em situações em que a linguagem e as práticas se configuram como matériasprimas da pesquisa, tem sido considerada apropriada para situações complexas em que há pouco conhecimento estruturado (Minayo, 2010; Minayo e Sanches, 1993), o que se aplica à realidade atual desses núcleos.

Essa questão também se expressa no alto índice de uso de dados primários, que são especialmente produzidos diretamente com os profissionais do Nasf. Além disso, a falta de informações quantitativas sistematizadas acerca das atividades desenvolvidas por esses núcleos, geralmente ocasionada pela 
reduzida disponibilidade de instrumentos de gestão adequados para o acompanhamento do trabalho, faz com que os dados secundários sejam pouco utilizados nessas pesquisas.

Em relação ao conteúdo das atividades identificadas nos diferentes artigos analisados, é notório o predomínio do cunho assistencial nas atividades de prevenção, de planejamento e gestão, e de promoção da saúde. Ainda é necessária a realização de estudos específicos para se afirmar que tais atividades têm efetivamente prevalecido sobre as demais nos diferentes territórios, mas a presente pesquisa registra indícios de que as ações clínico-assistenciais específicas têm ocupado lugar central na atuação do Nasf. Ao mesmo tempo que pode representar um avanço no cuidado oferecido aos sujeitos no espaço da APS (Ayres, 2004), isso pode significar também a restrição da integralidade da atenção, uma vez que a centralidade das ações clínico-assistenciais específicas, centradas no atendimento dos casos de danos à saúde, leva à secundarização de ações voltadas para os determinantes e os riscos (Teixeira e Vilasbôas, 2014). Ademais, considerando-se que dentre tais atividades prevalecem os atendimentos clínicos ambulatoriais, compartilhados entre profissionais ou não, sua hegemonia torna imperativo identificar as características da clínica que vem sendo construída no Nasf, o que inclui analisar os instrumentos utilizados no cuidado e as relações estabelecidas entre profissionais e entre estes e os usuários. Nesse particular, cabe ressaltar que a proposta de clínica ampliada (Campos e Amaral, 2007; Cunha, 2005), embora tida como referência para o trabalho do Nasf (Brasil, 2009), é pouco conhecida pelos profissionais, e pode estar sendo reproduzida a clínica tradicional fragmentada dos serviços especializados, de forma precarizada, visto que a estrutura física da APS não é adequada a tal finalidade, o que representaria um importante risco para o fortalecimento da ESF.

As atividades de cunho preventivo também se destacam no trabalho do Nasf e, embora menos representativas no conjunto, configuram as principais ações coletivas desses núcleos nos territórios. Nesse contexto, pode-se perceber a tendência a se reproduzir uma perspectiva tradicional de educação em saúde na APS, vertical e normatizadora, centrada na mudança de comportamentos e atitudes, uma vez que palestras e salas de espera se sobressaem em relação às demais atividades, o que contraria a perspectiva dialógica de prática educativa, emancipatória e participativa, que busca a transformação de saberes e a autonomia dos sujeitos (Machado et al., 2007; Alves, 2005). Dada a importância de atividades preventivas no trabalho do Nasf, torna-se primordial a qualificação dos profissionais para esta finalidade.

Em relação às atividades de planejamento e gestão, percebe-se a importante faceta do apoio institucional que o Nasf fornece aos processos de gestão da saúde, corroborando Moura e Luzio (2014), uma vez que novos processos organizacionais são construídos a partir dessa nova experiência de trabalho. 
Todavia, é importante ressaltar a sobrecarga de trabalho que isso pode representar, o que precisa ser melhor discutido em novas pesquisas acerca do tema.

Ademais, as atividades de organização do próprio trabalho também são significativas no cotidiano do profissional do Nasf, e embora o insuficiente detalhamento destas atividades dificulte a identificação da perspectiva de planejamento que as orienta, é possível perceber o privilégio a ações de cunho normativo e burocrático, contrariando a perspectiva estratégico-situacional que tem sido proposta para o SUS, cujo propósito é explicar, de maneira abrangente, situações e formas de intervenção sobre os problemas, de modo a resolvê-los ou controlá-los (Teixeira, 2010). Verifica-se também a reduzida institucionalização da avaliação do trabalho, revelando o espaço marginal que a avaliação ocupa nos serviços de saúde, ainda que cada vez mais seja reforçada a necessidade de se avaliar permanentemente as intervenções realizadas (Medina et al., 2005).

Igualmente, percebe-se que as ações voltadas para a análise da situação de saúde da população são pouco significativas, o que demonstra a limitada importância que se dá ao reconhecimento das necessidades de saúde da população coberta pelos Nasfs e contraria diversos autores que afirmam a importância destas para a organização e definição do trabalho em saúde (Paim e AlmeidaFilho, 2014; Teixeira, 2010; Cecílio, 2006). Nesse contexto, recomenda-se a realização de novos estudos em que se procure analisar com profundidade essas questões, sobretudo a perspectiva do planejamento no Nasf.

Pode-se inferir também que o lugar da promoção da saúde e da atuação intersetorial sobre o território tem sido, de forma geral, negligenciado no Nasf, aspecto particularmente preocupante, uma vez que estas atividades estão na base de propostas alternativas ao modelo vigente de atenção à saúde, como é caso da proposta da promoção e vigilância da saúde (Teixeira, 2006). O lugar marginal que estas atividades ocupam pode indicar certa fragilidade da proposta Nasf, sobretudo em relação à promoção da saúde, já que não foi possível identificar se todas estas atividades, comumente assumidas como de promoção da saúde, efetivamente se diferenciam daquelas voltadas para a prevenção. Este é um debate importante que vem sendo provocado por diversos autores (Pellegrini Filho, Buss e Esperidião, 2014; Czeresnia, 2003), e que precisa ser mais bem compreendido na realidade do Nasf.

Da mesma forma, as atividades específicas de capacitação dos profissionais da ESF foram pouco referidas nos artigos. Essa constatação, particularmente relevante quando se considera que o apoio matricial foi definido como eixo do trabalho no Nasf, precisa ser explorada em outros estudos, pois há pouca clareza em relação aos espaços em que ocorrem prioritariamente tais atividades, que podem ser as discussões de casos durante as reuniões com as eSF, as atividades tradicionais de formação como palestras e até mesmo os atendimentos ou visitas domiciliares compartilhadas. 
Finalizando os achados desta pesquisa, a preponderância de dificuldades sobre as facilidades expressa o quão desafiador tem sido implantar e desenvolver uma proposta tecnológica e politicamente inovadora no contexto do SUS, como é o caso do Nasf. Ainda assim, pode-se perceber que o perfil proativo, a boa relação entre os profissionais do Nasf e a estrutura organizacional contra-hegemônica que orienta sua proposta, baseada em trabalho matricial em equipe e não em atendimento ambulatorial, são elementos que convergem para o bom desempenho do trabalho em saúde nos territórios. Os documentos que normatizam a proposta dos Nasfs parecem atuar como um fator de proteção contra a pressão, interna ou externa, que os profissionais sofrem para estabelecer o modelo ambulatorial tradicional. Dessa forma, quando buscam construir novas práticas, esses profissionais contam com uma base que possibilita algum exercício de autonomia e criatividade, ainda que pouco específica em relação ao fazer cotidiano.

Assim, nota-se que o principal dificultador do trabalho no Nasf está relacionado à problemática do modelo de atenção, mais precisamente à hegemonia do modelo médico-assistencial na ESF, o que é observado tanto em situações de resistência das equipes à proposta não ambulatorial de trabalho do Nasf quanto em situações em que o próprio Nasf reproduz este modelo em seu interior. Essa constatação demonstra como a lógica produtivista de atendimento clínico-ambulatorial mantém fortalecida na ESF, em detrimento de ações de prevenção e promoção da saúde. Dessa forma, não parece ser possível o pleno funcionamento do Nasf em contextos onde a ESF não funciona como tal. É necessário analisar conflitos dessa natureza, assim como a desigualdade do poder entre equipes e profissionais, frequente no trabalho em saúde, o que dificulta o desenvolvimento de propostas alternativas (Villa et al., 2015; Oliveira, Moretti-Pires e Parente, 2011; Araújo e Rocha, 2007), buscando-se identificar possíveis contribuições e limites do Nasf para a reorientação da lógica hegemônica.

A falta de apoio da gestão e as más condições de trabalho também se destacam como importantes dificultadores do trabalho no Nasf, e revelam que o planejamento dos municípios não tem se mostrado suficiente para a efetivação da proposta. Muitas vezes focados no incentivo financeiro do MS para a implantação do Nasf, vê-se pouco investimento, sobretudo político, na proposta, o que fragiliza as diferentes experiências construídas. Essa situação também é observada no contexto geral do sistema de saúde, no qual a precariedade das redes de atenção à saúde faz com que o trabalho do Nasf seja redirecionado para dar conta da demanda reprimida por serviços especializados, questão central que precisa ser enfrentada para o fortalecimento da ESF (Assis, 2015). 


\section{Considerações finais}

A análise da produção científica sobre o Nasf, embora tenha revelado o crescente interesse dos pesquisadores da área da saúde coletiva sobre o tema, demonstra a necessidade de se expandir os estudos para diferentes regiões do país, dado o panorama de desigualdade no acesso à saúde e de fragmentação que ainda marca a estruturação do SUS, que se apresenta como uma arena permanente de conflitos e enfrentamentos entre distintos projetos de organização e gestão dos serviços (Santos e Campos, 2015; Teixeira, Souza e Paim, 2014; Andrade et al., 2013).

A análise do conteúdo dessa produção evidenciou uma tendência à reprodução da perspectiva assistencial hegemônica no âmbito do Nasf, o que reforça a permanência e a reprodução de um modelo de atenção à saúde centrado na prática clínica e no cuidado de doentes, em detrimento de um processo de mudança que enfatize a promoção da saúde e a prevenção de riscos e agravos. Esta análise permite afirmar que tal tendência decorre da existência de uma série de elementos dificultadores do trabalho nesse núcleo, tais como a aparente inadequação do perfil profissional, a falta de apoio da gestão e a manutenção de condições de trabalho não condizentes com a mudança do conteúdo e da organização das práticas profissionais, o que afeta sua articulação com as atividades das eSF e fortalece o modelo médico-assistencial centrado na atenção individual em detrimento de ações de promoção da saúde e de prevenção de riscos e agravos.

Esse panorama geral evidencia a necessidade de análises mais detalhadas sobre o processo de trabalho em saúde no âmbito do Nasf, com maior aprofundamento teórico, de forma a tornar possíveis o reconhecimento das potencialidades e dos limites desta proposta e a produção de meios para a transformação do modelo de atenção à saúde no SUS. Nesse sentido, sugerese a realização de estudos que incluam, além dos profissionais diretamente envolvidos com as atividades assistenciais, os demais atores envolvidos no trabalho do Nasf, como gestores, profissionais das eSF e usuários, de modo que diferentes pontos de vista contribuam para as análises das práticas de saúde e da conformação de modelos organizacionais que incluem o Nasf nas redes de atenção.

Do mesmo modo, é urgente a construção e aperfeiçoamento de instrumentos de gestão que possibilitem a síntese das atividades desenvolvidas no Nasf, com informação qualificada que considere sobretudo as características específicas desse trabalho. A implantação recente do sistema E-SUS pode significar uma mudança nesse quadro, uma vez que há instrumentos que viabilizam a coleta e a organização das informações a serem potencializados. 
Todas essas questões devem, necessariamente, ser atravessadas pela preservação da perspectiva transformadora da APS, de modo que as facilidades encontradas neste estudo sejam potencializadas e possam contribuir para a superação dos limites e favorecer a construção de uma proposta essencialmente inovadora, capaz de consolidar resultados coerentes com objetivos estratégicos da ESF no SUS.

\section{Colaboradores}

Vladimir Andrei Rodrigues Arce participou da concepção, levantamento de dados, análise, redação, revisão e finalização do artigo. Carmen Fontes Teixeira participou da concepção, revisão de conteúdo e finalização do artigo.

Resumen Este artículo tiene como objetivos sintetizar las características de la producción científica brasileña acerca de las actividades realizadas por los profesionales de los Núcleos de Apoyo a la Salud de la Familia, analizar el contenido de dichas actividades e identificar los aspectos facilitadores y dificultadores del proceso de trabajo del equipo. Se trata de una revisión de la literatura publicada en el período entre 2008 y 2015 en las bases de datos SciELO y Lilacs. Los resultados demuestran el predominio de estudios cualitativos que describen las actividades realizadas por profesionales específicos, especialmente la recepción, asistencia y rehabilitación, siendo menos expresivas las acciones de promoción de la salud, prevención, protección y vigilancia, planificación, programación, control, evaluación y gestión. Las acciones de capacitación y educación permanente son las menos discutidas. La proactividad y la buena relación interprofesional son aspectos facilitadores, mientras que la persistencia del modelo biomédico en el ámbito de la Salud de la Familia, las precarias condiciones de trabajo y la falta de apoyo de la gestión son aspectos dificulta $\neg$ dores. Se observa una tendencia a la reproducción de la perspectiva asistencial hegemónica en el ámbito del Núcleo de Apoyo a la Salud de la Familia, en lugar de un proceso de cambio orientado a la integralidad de la atención.

Palabras clave salud de la familia; atención primaria de la salud; práctica de la salud pública; políticas públicas de salud; equipo de asistencia al paciente. 


\section{Notas}

${ }^{1}$ Universidade Federal da Bahia, Instituto de Saúde Coletiva, Salvador, Bahia, Brasil. <vladimir.arce@ufba.br>

Correspondência: Universidade Federal da Bahia, Instituto de Ciências da Saúde, Departamento de Fonoaudiologia, Avenida Reitor Miguel Calmon s/n, CEP 40110-902, Vale do Canela, Salvador, Bahia, Brasil.

${ }^{2}$ Universidade Federal da Bahia, Instituto de Saúde Coletiva, Salvador, Bahia, Brasil. <carment@ufba.br>

\section{Referências}

ALMEIDA-FILHO, Naomar. Saúde como campo de práticas. In: ALMEIDA-FILHO, Naomar. $O$ que é saúde?. Rio de Janeiro: Editora Fiocruz, 2011. p. 105-139. (Temas em Saúde).

ALVES, Vânia S. Um modelo de educação em saúde para o Programa Saúde da Família: pela integralidade da atenção e reorientação do modelo assistencial. Interface: Comunicação, Saúde, Educação, Botucatu, v. 9, n. 16, p. 39-52, 2005.

ANDRADE, Mônica V. et al. Desigualdade socioeconômica no acesso aos serviços de saúde no Brasil: um estudo comparativo entre as regiões brasileiras em 1998 e 2008. Economia Aplicada, Ribeirão Preto, v. 17, n. 4, p. 623-645, 2013.

AQUINO, Rosana et al. Estratégia Saúde da Família e reordenamento do sistema de serviços de saúde. In: PAIM, Jairnilson S.; ALMEIDA-FILHO, Naomar. Saúde coletiva: teoria e prática. Rio de Janeiro: Medbook, 2014. p. 353-371.

ARAÚJO, Marize B. S.; ROCHA, Paulo M. Trabalho em equipe: um desafio para a consoli- dação da estratégia de saúde da família. Ciência \& Saúde Coletiva, Rio de Janeiro, v. 12, n. 2, p. 455-464, 2007.

ARCE, Vladimir A. R.; TEIXEIRA, Carmen F. Práticas de saúde e modelo de atenção no âmbito do Núcleo de Apoio à Saúde da Família em Salvador (BA). Saúde em Debate, Rio de Janeiro, v. 41, n. esp. 3, p. 228-240, 2017.

ASSIS, Marluce M. A. Redes de atenção à saúde e os desafios da Atenção Primária à Saúde: um olhar sobre o cenário da Bahia. In: ALMEIDA, Patty F.; SANTOS, Adriano M.; SOUZA, Mariluce K. B. Atenção primária à saúde na coordenação do cuidado em regiões de saúde. Salvador: Edufba, 2015. p. 45-64.

AYRES, José R. C. M. O cuidado, os modos de ser (do) humano e as práticas de saúde. Saúde e Sociedade, São Paulo, v. 13, n. 3, p. 16-29, 2004.

BARATA, Rita B. Condições de saúde da população brasileira. In: GIOVANELLA, Lígia et al. (orgs.). Políticas e Sistema de Saúde no Brasil. Rio de Janeiro: Editora Fiocruz, 2008. p. 167-213. 
BISPO JÚNIOR, José P.; MOREIRA, Diane C. Educação permanente e apoio matricial: formação, vivências e práticas dos profissionais dos Núcleos de Apoio à Saúde da Família e das equipes apoiadas. Cadernos de Saúde Pública, Rio de Janeiro, v. 33, n. 9, p. e00108116, 2017.

BRASIL, Ministério da Saúde. Portaria GM 154, de 24 de janeiro de 2008. Cria os Núcleos de Apoio à Saúde da Família - Nasf. Diário Oficial da União, Brasília, 25 jan. 2008. Seção 1, página 47-49. Disponível em: <http:// bvsms.saude.gov.br/bvs/saudelegis/gm/2008/ prt0154_24_01_2008.html>. Acesso em: 26 maio 2014.

BRASIL, Ministério da Saúde. Portaria GM n. 3.124, de 28 de dezembro de 2012. Redefine os parâmetros de vinculação dos Núcleos de Apoio à Saúde da Família (Nasf) Modalidades 1 e 2 às Equipes Saúde da Família e/ou Equipes de Atenção Básica para populações específicas, cria a Modalidade Nasf 3, e dá outras providências. Diário Oficial da União, Brasília, 29 dez. 2012. Disponível em: <http:// bvsms.saude.gov.br/bvs/saudelegis/gm/2012/ prt3124_28_12_2012.html>. Acesso em: 20 dez. 2017.

BRASIL. Ministério da Saúde. Secretaria de Atenção à Saúde. Departamento de Atenção Básica e Departamento de Ações Programáticas Estratégicas. Diretrizes do Nasf (Núcleo de Apoio a Saúde da Família). Brasília: Ministério da Saúde, 2009. (Cadernos de Atenção Básica, n. 27).

BRASIL. Ministério da Saúde. Cadastro Nacional de Estabelecimentos de Saúde (CNES). Datasus. Informações de Saúde. Rede Assistencial, 2016. Disponível em: < http://www2.datasus.gov.br/ DATASUS/index.php?area $=0204 \& i d=11676>$. Acesso em: 20 abr. 2016.

CAMPOS, Gastão W. S.; AMARAL, Márcia A. A clínica ampliada e compartilhada, a gestão democrática e redes de atenção como referenciais teórico-operacionais para a reforma do hospital. Ciência \& Saúde Coletiva, Rio de Janeiro, v. 12, n. 4, p. 849-859, 2007.

CAMPOS, Gastão W. S.; DOMITTI, Ana C. Apoio matricial e equipe de referência: Rio de Janeiro, uma metodologia para gestão do trabalho interdisciplinar em saúde. Cadernos de Saúde Pública, Rio de Janeiro, v. 23, n. 2, p. 399-407, 2007.

CECÍLIO, Luís C. O. As necessidades de saúde como conceito estruturante na luta pela integralidade e eqüidade na atenção em saúde. In: PINHEIRO, Roseni; MATTOS, Ruben A. (orgs.). Os sentidos da integralidade na atenção e no cuidado à saúde. Rio de Janeiro: IMS/ Uerj, 2006. p. 117-130.

CUNHA, Gustavo T. A construção da clínica ampliada na Atenção Básica. São Paulo: Hucitec, 2005.

CUNHA, Gustavo T.; CAMPOS, Gastão W. S. Apoio matricial e Atenção Primária em Saúde. Saúde e Sociedade, São Paulo, v. 20, n. 4, p. 961-970, 2011.

CONSELHO NACIONAL DE DESENVOLVIMENTO CIENTÍFICO E TECNOLÓGICO (CNPq). Censo do Diretório dos Grupos de Pesquisa no Brasil, 2014. Disponível em: <http://lattes. cnpq.br/web/dgp/por-uf2>. Acesso em: 15 jan. 2016.

CZERESNIA, Dina. O conceito de saúde e a diferença entre prevenção e promoção. In: CZERESNIA, Dina; FREITAS, Carlos M. (orgs.). Promoção da saúde: conceitos, reflexões, tendências. Rio de Janeiro: Editora Fiocruz, 2003. p. 39-53.

MACHADO, Maria F. A. S. et al. Integralidade, formação de saúde, educação em saúde e as propostas do SUS: uma revisão conceitual. Ciência \& Saúde Coletiva, Rio de Janeiro, v. 12, n. 2, p. 335-342, 2007.

MEDINA, Maria G. et al. Uso de modelos teóricos na avaliação em saúde: aspectos con- 
ceituais e operacionais. In: HARTZ, Zulmira M.A.; VIEIRA-DA-SILVA, Lígia M. Avaliação em saúde: dos modelos teóricos à prática na avaliação de programas e sistemas de saúde. Salvador: Edufba; Rio de Janeiro: Editora Fiocruz, 2005. p. 41-63.

MINAYO, Maria C. S. O desafio do conhecimento: pesquisa qualitativa em saúde. 12 ed. São Paulo: Hucitec; Rio de Janeiro: Abrasco, 2010.

MINAYO, Maria C. S.; SANCHES, Odécio. Quantitativo-qualitativo: oposição ou complementaridade? Cadernos de Saúde Pública, Rio de Janeiro, v. 9, n. 3, p. 239-248, 1993.

MOURA, Renata H.; LUZIO, Cristina A. O apoio institucional como uma das faces da função apoio no Núcleo de Apoio à Saúde da Família (Nasf): para além das diretrizes. Interface: Comunicação, Saúde, Educação, Botucatu, v. 18, supl.1, p. 957-970, 2014.

OLIVEIRA, Hadelândia M.; MORETTI-PIRES, Rodrigo O.; PARENTE, Rosana C. P. As relações de poder em equipe multiprofissional de saúde da família segundo um modelo teórico arendtiano. Interface: Comunicação, Saúde, Educação, Botucatu, v. 15, n. 37, p. 539-550, 2011.

OLIVEIRA, Mônica M.; CAMPOS, Gastão W. S. Apoios matricial e institucional: analisando suas construções. Ciência \& Saúde Coletiva, Rio de Janeiro, v. 20, n. 1, p. 229-238, 2015.

PAIM, Jairnilson S. O objeto e a prática da saúde coletiva: o campo demanda um novo profissional? In: PAIM, Jainilson S. Desafios para a saúde coletiva no século XXI. Salvador: Edufba, 2006. p. 99-116.

PAIM, Jairnilson S.; ALMEIDA-FILHO, Naomar. Análise da situação de saúde: o que são necessidades e problemas de saúde? In: PAIM, Jairnilson S.; ALMEIDA-FILHO, Naomar. Saúde coletiva: teoria e prática. Rio de Janeiro: Medbook, 2014. p. 3-12.
PAIM, Jairnilson S. et al. The Brazilian health system: history, advances, and challenges. Lancet, v. 377, n. 9.779, p. 1.778-1.797, 2011.

PEDUZZI, Marina. Equipe multiprofissional de saúde: conceito e tipologia. Revista de Saúde Pública, São Paulo, v. 35, n. 1, p. 103-109, 2001.

PEDUZZI, Marina; SCHRAIBER, Lília B. Processo de trabalho em saúde. In: ESCOLA POLITÉCNICA DE SAÚDE JOAQUIM VENÂNCIO (org.). Dicionário da educação e trabalho em saúde. Rio de Janeiro: EPSJV, 2006. p. 199-207.

PELLEGRINI FILHO, Alberto; BUSS, Paulo M.; ESPERIDIÃO, Monique A. Promoção da saúde e seus fundamentos: determinantes sociais da saúde, ação intersetorial e políticas saudáveis. In: PAIM, Jairnilson S.; ALMEIDA FILHO, Naomar. Saúde coletiva: teoria e prática. Rio de Janeiro: Medbook, 2014. p. 305-326.

REIS, Marcos A. S. et al. A organização do processo de trabalho em uma unidade de saúde da família: desafios para a mudança das práticas. Interface: Comunicação, Saúde, Educação, Botucatu, v. 11, n. 23, p. 655-66, 2007.

RIBEIRO, Edilza M.; PIRES, Denise; BLANK, Vera L. A teorização sobre processo de trabalho em saúde como instrumental para análise do trabalho no Programa Saúde da Família. Cadernos Saúde Pública, Rio de Janeiro, v. 20, n. 2, p .438-446, 2004.

SANTOS, Lenir; CAMPOS, Gastão W. S. SUS Brasil: a região de saúde como caminho. Saúde e Sociedade, São Paulo, v. 24, n. 2, p. 438446, 2015.

SHIMIZU, Helena E.; CARVALHO JUNIOR, Daniel A. O processo de trabalho na Estratégia Saúde da Família e suas repercussões no processo saúde-doença. Ciência \& Saúde Coletiva, Rio de Janeiro, v. 17, n. 9, p. $2.405-$ 2.414, 2012. 
TEIXEIRA, Carmen F. Saúde da família, promoção e vigilância: construindo a integralidade da atenção à saúde no SUS. In: TEIXEIRA, Carmen F; SOLLA, Jorge P. Modelo de atenção à saúde: promoção, vigilância e saúde da família. Salvador: Edufba, 2006. p. 59-83.

TEIXEIRA, Carmen F. (org.). Planejamento em saúde: conceitos, métodos e experiências. Salvador: Edufba, 2010.

TEIXEIRA, Carmen F.; VILASBÔAS, Ana L. Q. Modelos de atenção à saúde no SUS: mudança ou conservação? In: PAIM, Jairnilson S.; ALMEIDA-FILHO, Naomar. Saúde coletiva: teoria e prática. Rio de Janeiro: Medbook, 2014. p. 287-301.

TEIXEIRA, Carmen F.; SOUZA, Luís E. P. F.; PAIM, Jairnilson S. Sistema Único de Saúde
(SUS): a difícil construção de um sistema universal na sociedade brasileira. In: PAIM, Jairnilson S.; ALMEIDA-FILHO, Naomar. Saúde coletiva: teoria e prática. Rio de Janeiro: Medbook, 2014. p. 3-12.

VIANA, Ana L. Abordagens metodológicas em políticas públicas. Revista de Administração Pública, Rio de Janeiro, v. 30, n. 2, p. 5-43, 1996.

VILLA, Eliana A. et al. As relações de poder no trabalho da Estratégia Saúde da Família. Saúde em Debate, Rio de Janeiro, v. 39, n. 107, p. 1.044-1.052, 2015.

VOSGERAU, Dilmeire S. R.; ROMANOWSKI, Joana P. Estudos de revisão: implicações conceituais e metodológicas. Diálogo Educacional, Curitiba, v. 14, n. 41, p. 165-189, 2014.

Recebido em 25/08/2017.

Aprovado em 13/03/2018. 